\title{
Solvent evaporation driven entrapment of magnetic nanoparticles in mesoporous frame for designing a highly efficient MRI contrast probe
}

\author{
K. Saikia ${ }^{1}$, K. Bhattacharya ${ }^{2,8}$, D. Sen ${ }^{3}$, S. D. Kaushik ${ }^{4}$, J. Biswas ${ }^{5}$, S. Lodha ${ }^{5}$, B. Gogoi ${ }^{6}$,
} A.K. Buragohain ${ }^{6,7}$, W. Kockenberger ${ }^{8}$ and P. Deb ${ }^{1 *}$

1 Advanced Functional Material Laboratory (AFML), Department of Physics, Tezpur University

(Central University), Tezpur 784028, India.

2 Atomic Energy and Alternative Energies Commission, Institut rayonnement matière de Saclay (IRAMIS), France

3 Solid State Physics Division, Bhabha Atomic Research Centre, Mumbai 400085, India.

4 UGC-DAE Consortium for Scientific Research, Mumbai Centre, R-5 Shed, BARC, Mumbai 400085, India

5 Department of Electrical Engineering, IIT Bombay, Mumbai 400076, India

6 Department of Molecular Biology and Biotechnology, Tezpur University, Napaam, Tezpur 784028, Assam, India

7 Dibrugarh University, Dibrugarh 786004, Assam, India

8 Sir Peter Mansfield Magnetic Resonance Centre, School of Physics and Astronomy, University of Nottingham, Nottingham NG7 2RD, UK

*Corresponding author's E-mail ID: pdeb@tezu.ernet.in

\begin{abstract}
The present work reports a novel strategy of assembling maghemite $\left(\gamma-\mathrm{Fe}_{2} \mathrm{O}_{3}\right)$ nanoparticles (NPs) in mesoporous silica host for developing a highly efficient MRI contrast probe. Shrinkage of hydrophobic environment due to the continuous evaporation of chloroform from Chloroform-in-Water emulsions pushes the hydrophobic $\gamma-\mathrm{Fe}_{2} \mathrm{O}_{3}$ NPs towards the hydrophobic pores of silica spheres resulting in a water soluble dense assembly structure. Mesoporous silica only with straight pores is found to be suitable for this particular entrapment process, while with curved and twisted pores, NPs are found to be seated on the surface only. So-developed assembly system has retained the superparamagnetic behaviour of its comprising NPs and exhibited high colloidal stability and biocompatibility. A significant
\end{abstract}


enhancement in MRI transverse relaxivity to $386.2 \mathrm{mM}^{-1} \mathrm{~s}^{-1}$ from $191.8 \mathrm{mM}^{-1} \mathrm{~s}^{-1}$ of isolated primary $\gamma-\mathrm{Fe}_{2} \mathrm{O}_{3}$ NPs, has been obtained due to the strong magnetic field generated by the large number of NPs packed in the porous channels and consequent faster relaxation process. The fabrication strategy can be extended for the development of designed secondary nanostructures with new magnetic effects and physical properties.

KEY WORDS: solvent evaporation, magnetic NPs, MRI contrast agent, mesoporous silica, assembly of NPs

\section{Introduction}

Directed assembling of magnetic NPs has been emerged as a promising bottom-up approach for designing their secondary nanostructures with controllable shape, size and morphology The key in the creation of these unique nanostructures is the controlled assembling of the building blocks. This new class of nanostructured materials has high sensitivity and can overcome the limitations of nanoscale single domain systems, making them highly desired components for designing smart sensors, intelligent systems, and adaptive structures [1-3]. Application potential of these assembled magnetic NPs systems as the MRI contrast enhancers in the field of bio-imaging research has attracted lot of research attentions in the recent time. Ferrite based superparamagnetic NPs are already established as the highly efficient $\mathrm{T}_{2}$ or dark contrast enhancers in Magnetic Resonance Imaging (MRI) and among them, NPs of magnetite $\left(\mathrm{Fe}_{3} \mathrm{O}_{4}\right)$ and maghemite $\left(\gamma-\mathrm{Fe}_{2} \mathrm{O}_{3}\right)$ are highly promising due to their higher saturation magnetizations [4]. However, the weak magnetic trackability due to the high Brownian motion of these fine sized NPs hampers on their potential for biomedical applications. Recent works in this direction showed that a compact dense structure of the superparamagnetic NPs, i.e. formation of their secondary nanostructures can short out this 
problem and additionally enhance MRI relaxivity by aggregation-induced effect [5]. This type of secondary nanostructures can be obtained usually by employing three different basic bottom-up approaches; (i) single step process by optimizing the concentration of surfactants, where the nucleated primary particles follow the secondary growth process, (ii) by modulating the intermolecular packing parameter of participating self-assembling amphiphilic molecules and (iii) by using suitable host structures [6, 7]. However, it is important that the used capping molecules or support materials should not limit the penetration of water molecules to the core of the comprising magnetic NPs, so that it will not impart negative effect on MRI contrast efficiency [8].

Submicron size silica grains with porous architecture have shown tremendous opportunities in this direction. Their high chemical stability, bio-compability, versatility with respect to surface functionalization, and most importantly their versatile porous networks and the ability of labelling with wide range of functional molecules make them a suitable host material for fabrication of secondary magnetic nanostructures [9]. This host material also takes account of ensuring water accessibility to core of the entrapped magnetic NPs by its porous channels. In few works, silica was employed as the shell material for encapsulating clusters of magnetic NPs, while in some cases silica was employed as the core structure on which magnetic NPs are grafted. For example, encapsulation of magnetic NPs by mesoporous silica as a result of hydrolysis and condensation of silica precursors (TESO) in presence of different surfactants has been explored by many groups [10]. In another method, sonication of Fe $(\mathrm{CO})_{5}$ solution in decalin containing silica particles resulted in structures composed of iron oxide NPs heterogeneously grafted onto the silica spheres [11]. Another alternative strategy showed the coating of silica spheres with layers of iron compounds based on the forced hydrolysis of iron (III) acetylacetonate solutions containing the silica cores and a surfactant [12]. The coating of silica spheres with layers of preformed magnetite $\left(\mathrm{Fe}_{3} \mathrm{O}_{4}\right)$ NPs using a heterocoagulation 
process facilitated by charging the silica surface through the addition of a polyelectrolyte film has been also reported, although this method produced rough coatings [13]. Thus, in these types of structures either core or shell part of the silica spheres remains unutilized. In contrast, the present work demonstrates a mesoporous silica supported dense assembly structure of $\gamma-\mathrm{Fe}_{2} \mathrm{O}_{3} \mathrm{NPs}$, where, NPs get entrapped inside the silica spheres and also get sited on the surface of it. In this work, $\gamma-\mathrm{Fe}_{2} \mathrm{O}_{3} \mathrm{NPs}$ have been chosen over $\mathrm{Fe}_{3} \mathrm{O}_{4}$ NPs due to its higher physico-chemical stability. A facile approach was employed for developing this secondary nanostructure, where the NPs are entrapped homogenously inside the porous channels of silica spheres. The method is based on solvent evaporation mediated entrapment of NPs inside the porous network of silica spheres of submicron size. The developed magnetic assembly system can also have potential for being a multifunctional nanoprobe with other functionalities like targeted drug carrier along with the MRI contrast enhancer. Also, the process used for developing this structure can be further employed for developing new secondary nanostructures of other nanomaterials.

\section{Experimental section}

2.1 Materials: Tetraethyl orthosilicate (reagent grade, 98\%) was purchased from SigmaAldrich. Ammonium hydroxide $\left(\mathrm{NH}_{4} \mathrm{OH}\right)$, Iron sulphate $\left(\mathrm{FeSO}_{4} \cdot 7 \mathrm{H}_{2} \mathrm{O}, \quad 99 \%\right)$, tetramethylamonium hydroxide (TMAOH assay 25\%), oleic acid $\left(\mathrm{C}_{18} \mathrm{H}_{34} \mathrm{O}_{2}, 90 \%\right)$, dimethylsulphoxide (DMSO, $99 \%$ ) and cetyltrimethyl ammonium bromide (CTAB, 99\%), Ethyl acetate (EtoAC) were procured from Merck, India. All chemicals were used directly without further purification. Milli-Q water was used throughout all the reactions.

2.2 Synthesis of Mesoporous silica spheres (S1): The synthesis of the Mesoporous silica spheres has been performed using a modified Stöber method. Here, $0.01 \mathrm{~g}$ of CTAB was added to $100 \mathrm{ml}$ water and the $\mathrm{pH}$ of the solution was adjusted around 10.00 by adding $\mathrm{NH}_{4} \mathrm{OH}$ (25\% solution). Then, $1 \mathrm{ml}$ of the silica precursor solution, TEOS was added drop 
wise with the subsequent addition of $5 \mathrm{ml}$ ethyl acetate. The mixture solution instantly became turbid and white precipitation was observed. The obtained precipitate was washed with distilled water three times and dried overnight. The similar protocol was followed for the preparation of the other two silica samples S2 and S3. The only difference is that here 0.05 and $0.1 \mathrm{~g}$ of CTAB were added for S2 and S3 respectively.

2.3 Synthesis of iron oxide (IO) NPs: Oleic acid capped iron oxide NPs were synthesized by using a modified co-precipitation method as reported [14].

2.4 Synthesis of iron oxide NPs loaded silica spheres (S@IO): $50 \mathrm{mg}$ of the already prepared silica powder and $0.05 \mathrm{~g}$ of CTAB were mixed in $100 \mathrm{ml}$ water. A dispersion of iron oxide NPs prepared in $4 \mathrm{ml}$ chloroform was poured drop wise to the above solution and stirred vigorously for 30 minutes for the preparation of a homogenous emulsion solution. The obtained emulsion solution was kept at $60^{\circ} \mathrm{C}$ in an oven until the complete evaporation of the chloroform. The resultant brown colour solution was centrifuged to precipitate down the particles. The obtained precipitate was washed with water and dried overnight.

\section{Characterization details}

High resolution transmission electron microscopy (HRTEM) was performed using a JEOL 2100 electron microscope operated at an accelerating voltage of $200 \mathrm{kV}$. The particle size distribution was calculated on about 200 nanoparticles from multiple images in bright field mode and fitted with Gaussian function. Raman spectra were taken using a Renishaw system with $\mathrm{Ar}^{+}$laser excitation source of wavelength $514.5 \mathrm{~nm}$. The surface capping characteristics was characterized with Nicolet Fourier transforms infrared (FTIR) spectrometer. SAXS experiments were performed using a laboratory based SAXS instrument. The sample to detector distance was $\sim 1 \mathrm{~m}$. The radial averaged scattering profiles were obtained a function of wave-vector transfer (q). The dynamic light scattering (DLS) and Zeta potential 
measurements were performed using a NanoPlus-3, Nano Particle Analyzer, Micromeritics instrument at room temperature. Thermogravimetric analysis (TGA) was carried out using a thermogravimetric analyzer (TGA-50, Shimadzu), where, the samples were heated in $\mathrm{N}_{2}$ atmosphere with flow rate of $20 \mathrm{ml} / \mathrm{min}$. Magnetic measurements such as $\mathrm{M}-\mathrm{H}$ and M-T with ZFC and FC protocols were carried out using 9T Quantum Design Physical Property Measurements system (PPMS) equipped with a vibrating sample magnetometer (VSM). XPS characterizations were performed using an X-ray photo spectrometer with monochromatic $\mathrm{Al}$ $\mathrm{K} \alpha$ source $(\mathrm{hv}=1486.6 \mathrm{eV})$. For MRI transverse relaxivity measurements, stable suspensions of synthesized maghemite NPs and their assembly at predetermined concentrations in agarose water were filled in standard NMR tubes $(5 \mathrm{~mm}$ diameter) and introduced inside a $9.4 \mathrm{~T}$ NMR spectrometer. Agarose was used to stabilise the suspension and to prevent the NPs from sedimentation during the experiments. Seven different concentrations of NPs were used and an additional reference tube that only contained the water agarose medium. For measuring $\mathrm{T}_{2}$, multi echo experiments were carried out with spatial resolution (field of view of $25 \times 25 \mathrm{~mm}$, image matrix of $128 \times 128$ pixels and a slice thickness of $1 \mathrm{~mm}$ ) providing a set of $\mathrm{T}_{2}$-weighted images. Data analysis was carried out by importing the time data into a MATLAB programme. Regions of interest (ROI, 10x10 pixels) were manually selected in such a way that they coincide with the cross sections of the NMR tubes in the images and the average signal intensity in each ROI was calculated and its attenuation with increasing number of echoes determined by fitting a simple exponential decay function. $\mathrm{T}_{2}$ weighted images were acquired at a fixed repetition time and at variable echo times. In this manner for each concentration of particles a transverse relaxation $\mathrm{T}_{2}$ time constant was obtained. Its inverse, the transversal relaxation rate $\mathrm{R}_{2}$, was plotted against the corresponding concentration and by linear fitting the transversal relaxivity $r_{2}$ was calculated. 


\section{Results and discussion}

\subsection{Entrapment of the $\gamma-\mathrm{Fe}_{2} \mathrm{O}_{3} \mathrm{NPs}$ in silica}

The uniform size of around $4.9 \mathrm{~nm}$ and well separation of the oleic acid stabilized iron oxide NPs employed for entrapping in mesoporous silica sphere is shown in the Fig.1a (i). TEM images of the silica spheres before and after NPs' entrapments are shown in the Figs. 1a (ii) and 1a (iii) respectively, where it is observed that the silica spheres with hexagonally packed straight pores have been densely filled up by the NPs. Fig. 1a (iv) shows a magnified view of the entrapped NPs in silica a sphere.

(a)
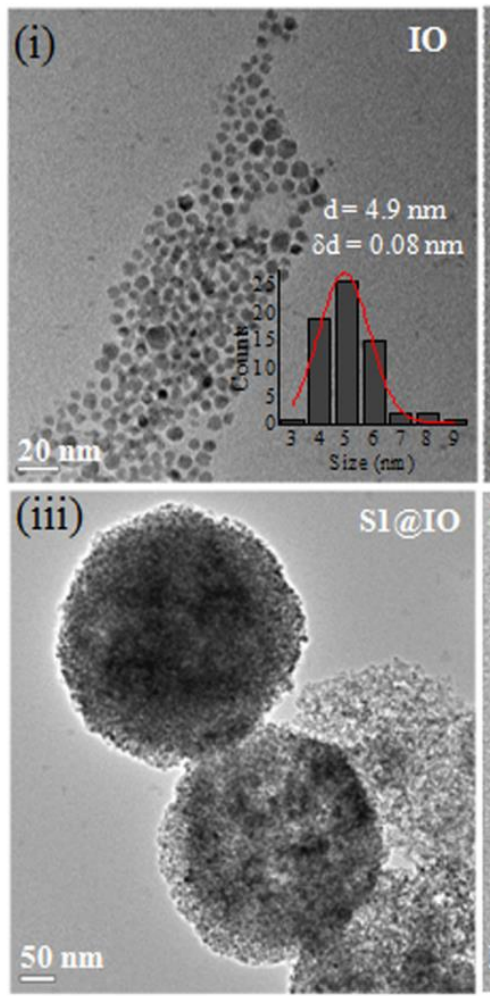

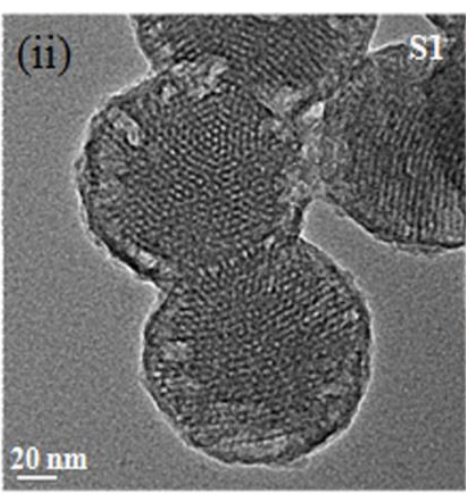

(iv)

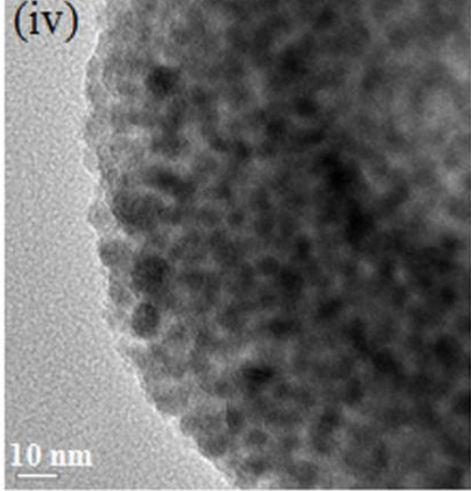

(b)

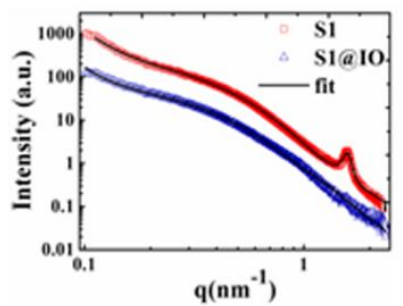

(c)

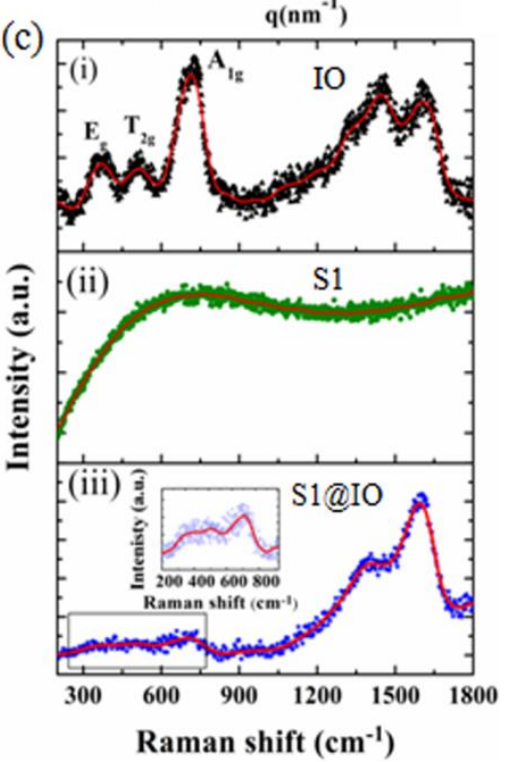

Fig. 1 (a) TEM images of iron oxide NPs (i), mesoporous silica spheres (ii), IO loaded silica sphere (iii), magnified view of the assembly system (iv); (b) the comparative SAXS plots (in double logarithmic scales) of mesoporous silica (S1) and IO loaded silica (S1@ IO) systems and (c) Raman spectra of IO NPs (i), S1 (ii) and) S1@IO (iii). The inset of Fig.c (iii) shows 
the characteristic peaks of $\gamma-\mathrm{Fe}_{2} \mathrm{O}_{3}$ in the $\mathrm{S} 1 @ \mathrm{IO}$ system. The inset of the Fig.1 (a) shows the size distribution plot of the IO nanoparticles best fitted with Gaussian distribution function.

No separate aggregation of the NPs is observed on outer part of the silica spheres. A low magnified image shown in supplementary Fig. s1 justifies the uniform occurrence of this type of assembly system. The effective entrapment of the iron oxide NPs in the pores of the silica system is further confirmed from the complete suppression of the characteristic ordering peak in the SAXS plot (shown in the Fig. 1b). This implies that the entrapment of the iron oxide NPs breaks the hexagonal pore periodicity of silica in the S1 sample. The SAED (shown in supplementary Fig.s2) pattern taken in the magnetic assembly system shows the characteristic diffraction rings of face centred cubic structure of maghemite $\left(\gamma-\mathrm{Fe}_{2} \mathrm{O}_{3}\right)$ (JCPDS Card 391346) [15]. The intrinsic maghemite $\left(\gamma-\mathrm{Fe}_{2} \mathrm{O}_{3}\right)$ phase of iron oxide NPs and retaining of the same phase in the assembly system have been further confirmed from the observed characteristic peaks of maghemite for both the samples in Raman spectra (shown in the Fig.1c). The peaks at around $712 \mathrm{~cm}^{-1}, 510 \mathrm{~cm}^{-1}$ and $360 \mathrm{~cm}^{-1}$ are attributed to the $\mathrm{A}_{2 \mathrm{~g}}, \mathrm{~T}_{2 \mathrm{~g}}$ and $E_{2 g}$ modes of maghemite phase respectively $[16,17]$. The broad peaks at around 1452 $\mathrm{cm}^{-1}$ and $1611 \mathrm{~cm}^{-1}$ can be due to the disordered carbon chain of oleic acid molecules capped over the particles $[18,19]$. It is observed that the pure silica sample has not shown any characteristic Raman peaks. The intensities of all the characteristic maghemite peaks have been diminished significantly for the assembly system, which is obvious due to the entrapment of the particles inside the pores. However, the assembly system has also shown the broad peaks of the disordered carbon chain, but with relatively higher intensities. This might be due to the additional contribution of carbons of CTAB molecules on the assembly system. To have an understanding of the process of entrapment, another two silica host systems (S2 and S3) with different pore morphologies have also been prepared and tried to develop the similar assembly systems by employing the similar protocol. However, unlike 
the first case, no signature of entrapment of the NPs has been observed here. In contrast, here NPs are observed to be aggregated separately on the surface of the silica grains (shown in Fig.2a (iii) and Fig.2a (iv)). TEM images show that with the increasing concentration of CTAB the pore morphology of the silica has been changed from straight to curved and then to twisted one (as shown in the Fig.1a(ii), Fig.2a (i) and Fig.2a (ii)). This variation in the pore morphology and also pore size can play important role in this type of entrapment process. To have a better insight, these later two pristine silica systems and also their respective NPs conjugated systems have been also characterized by Small-angle X-ray scattering (SAXS). SAXS plots in the Fig.2b (i) and Fig.2b (ii) show that the prominent peaks, corresponding to positional correlation among the pores, remain mostly unaffected even after the incorporation of NPs in the case of S2 and S3 samples. However, for the first system (S1), this peak is vanished completely as shown in the Fig.1b. At first instance, it can be argued that the size of the pores in the case of S2 and S3 samples are smaller and therefore, the particles are not going inside properly. However, the obtained pore size distribution (shown in Fig.3) has confirmed that the later two systems have even bigger pore sizes than the first one (S1). The SAXS profiles of the three pristine silica systems (S1, S2 and S3) have been fitted using the Porod cylinder form factor and Debye-Anderson-Brumberger (DAB) correlation function (equation 1), from which their pore size distribution and the pore morphologies have been derived (details of the fittings are provided in supplementary section s1 ).

$$
I(q)=I_{o} \frac{1}{\left[1+(q \xi)^{2}\right]^{2}}
$$

Where, $\xi$-Correlation length and $\mathrm{I}_{\mathrm{o}}$ is the forward scattering.

The obtained parameters have been shown in the supplementary Table s1 of, where it is observed that pore radius and pore lengths have been increased for the silica samples 
prepared with higher concentration of CTAB. An earlier work also showed that the increasing concentration of CTAB with constant TEOS concentration increases pore volume [20]. Interestingly, here it is also found that the effective pore lengths are larger than the average sizes of the silica grain for the S2 and S3 samples, which implies the folding nature of the pores. Therefore, the reasonable factors of not entrapping the particles by the later two silica systems can be either rigidity of the pores or bending/twisting nature of the pores. If the pores are rigid enough it cannot pull the bigger size particles inside it or the force felt by the particles during solvent evaporation (which is explained in the section 4.2) is not enough to promote the filling process. Another factor can be the roughness of the silica structures. In the first system, some bigger size random voids are observed which can act as the door for the particles to enter inside the pores. However, in the later two systems, such voids are not seen and the silica structures become smoother. The smoothness of these later two systems (S2 and S3) and the roughness of the first silica (S1) system can be further confirmed from their scattering plots. For the S2 and S3 samples, it is seen that the scattering profiles show a complete Porod behaviour, while the sample S1 shows a broad hump. The observed humps between $\mathrm{q}$ values 0.7 to $1 \mathrm{~nm}^{-1}$ before the characteristic ordering peak for the composite samples prepared with S2 and S3 silica hosts indicates the aggregation of the NPs outside of the silica structures. 
(a)
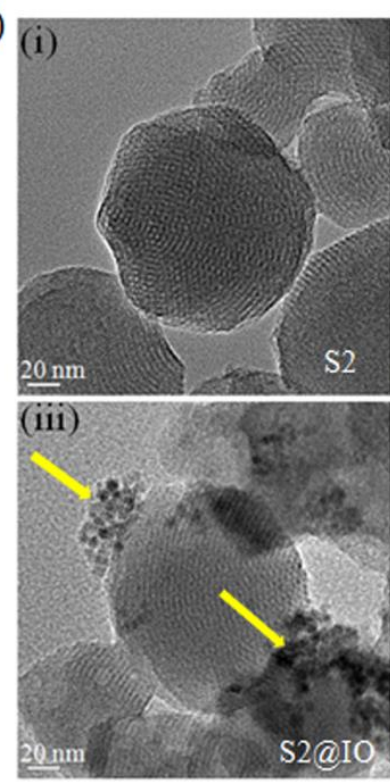

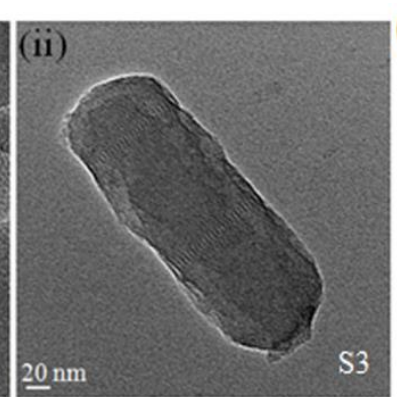

S3

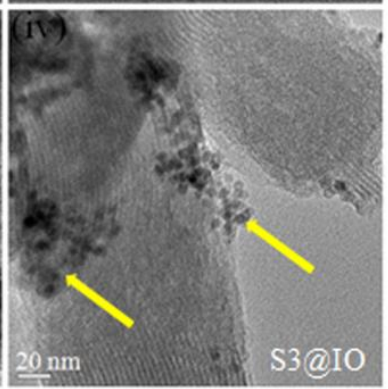

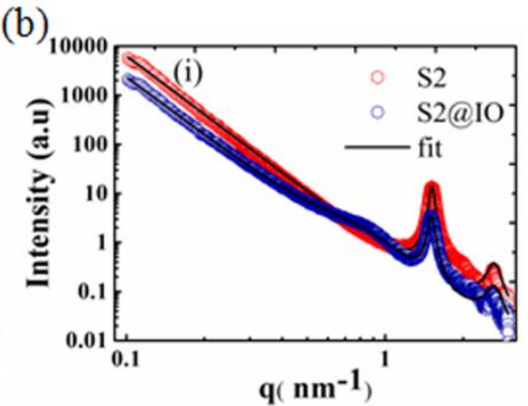

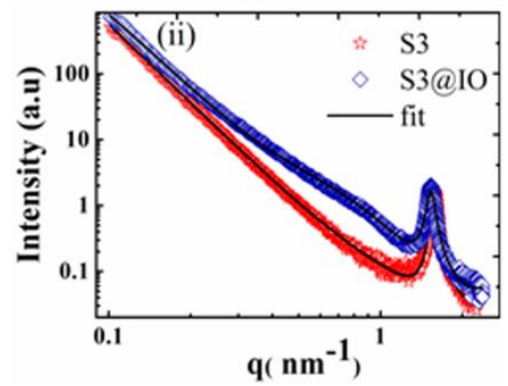

Fig.2 (a) TEM images of mesoporous silica with curved pores, S2 (i), and twisted pores, S3 (ii), IO conjugated S2, S2@IO (iii) and IO conjugated S3, S3@IO (iv); (b) comparative SAXS (in double logarithmic scale) plot of S2 and S2@IO (i) and S3 and S3@IO (ii). The yellow arrows on the TEM images are showing the aggregated IO nanoparticles on the surface of the silica structures.

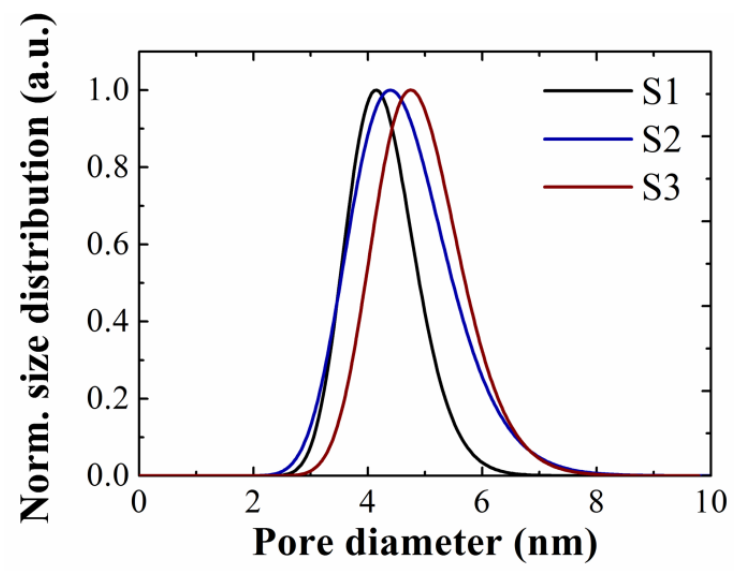

Fig.3 Pore size distribution plots of the S1, S2 and S3 samples obtained from the respective SAXS profile fittings. 


\section{2 Mechanism of the entrapment process}

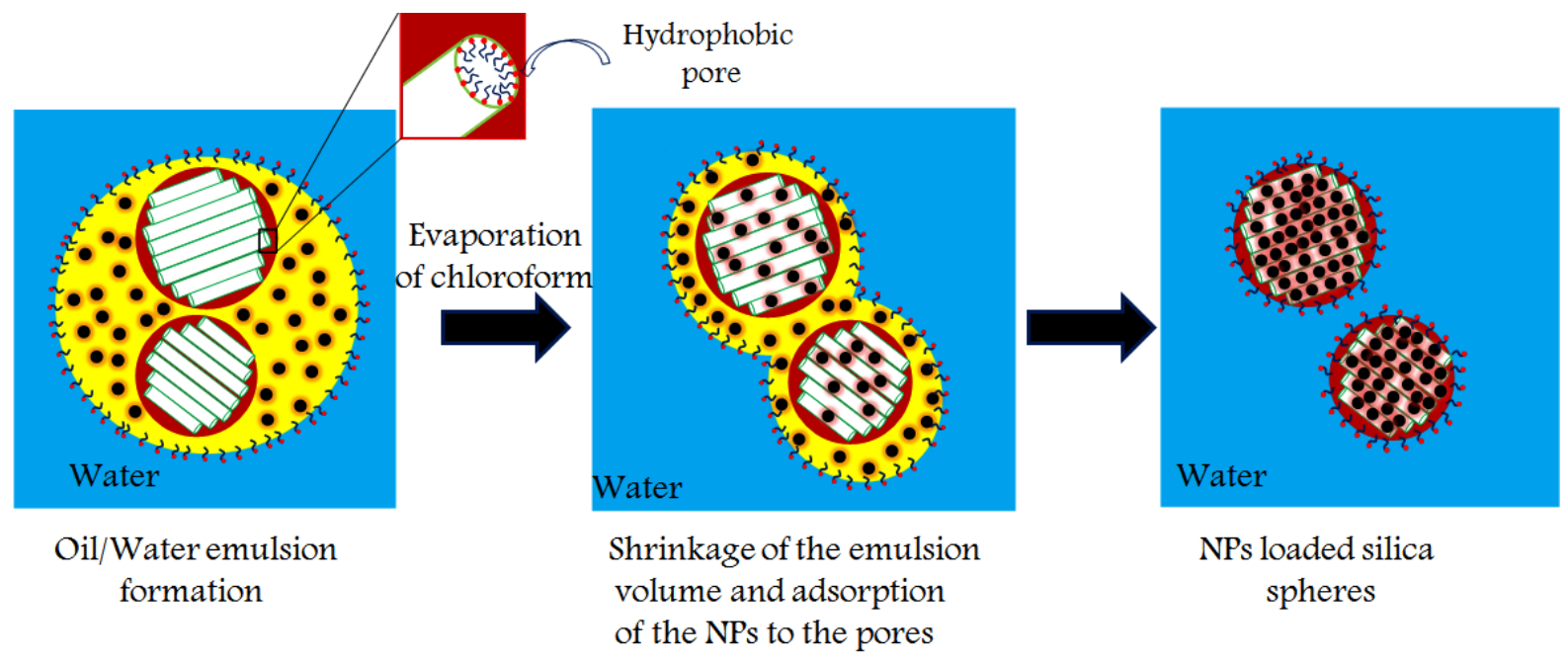

Fig. 4 A schematic of the entrapment mechanism of the IO NPs in mesoporous silica spheres. The inset on the figure shows the CTAB molecules inside the pores projecting their carbon chain outwards.

A plausible mechanism is presented for getting an idea of the entrapment process of the nanoparticles inside the pores (shown in Fig.4). When both the components, i.e. the NPs in chloroform and the suspension of silica spheres with CTAB were mixed, an Oil-in-Water emulsion solution was formed (optical micrographs of the formed emulsion system is shown in the supplementary Fig.s3. The formed emulsion is basically of chloroform droplets dispersed in water phase stabilized by the surfactant CTAB, where in the oil phase both IO nanoparticles and Silica spheres get dispersed as shown in the schematic. In general, silica particles are hydrophilic due to the high abundance of Silanol ( $\mathrm{Si}-\mathrm{OH})$ groups on their surface. However, here the as-prepared silica particles are hydrophobic in nature due to the dominancy of remaining CTAB molecules inside its pores. Hydrophobic nature of the silica system is shown the supplementary Fig.s3, where silica particles are observed to be easily dispersed in the chloroform phase. Therefore, here also the mesoporous silica particles are likely to be in chloroform of the developed Chloroform/Water emulsion system. As soon as 
the evaporation of the chloroform starts, hydrophobic NPs are bound to be absorbed in to the pores of the silica spheres due to the continuous shrinkage of the hydrophobic environment. It is also mentionable that the pores of the silica spheres have hydrophobic environment due to the presence of CTAB molecules projecting their carbon chain outwards. Therefore, there is a high probability of hydrophobic interaction between the NPs and the carbon chain of CTAB molecules, which eventually favours this adsorption process. As the evaporation process of the chloroform continues, NPs gradually enter to the pores of the silica spheres. CTAB molecules engaged for stabilizing the emulsion droplets also envelope the clusters after completion of the evaporation process.

\section{3 DLS and Zeta potential studies}
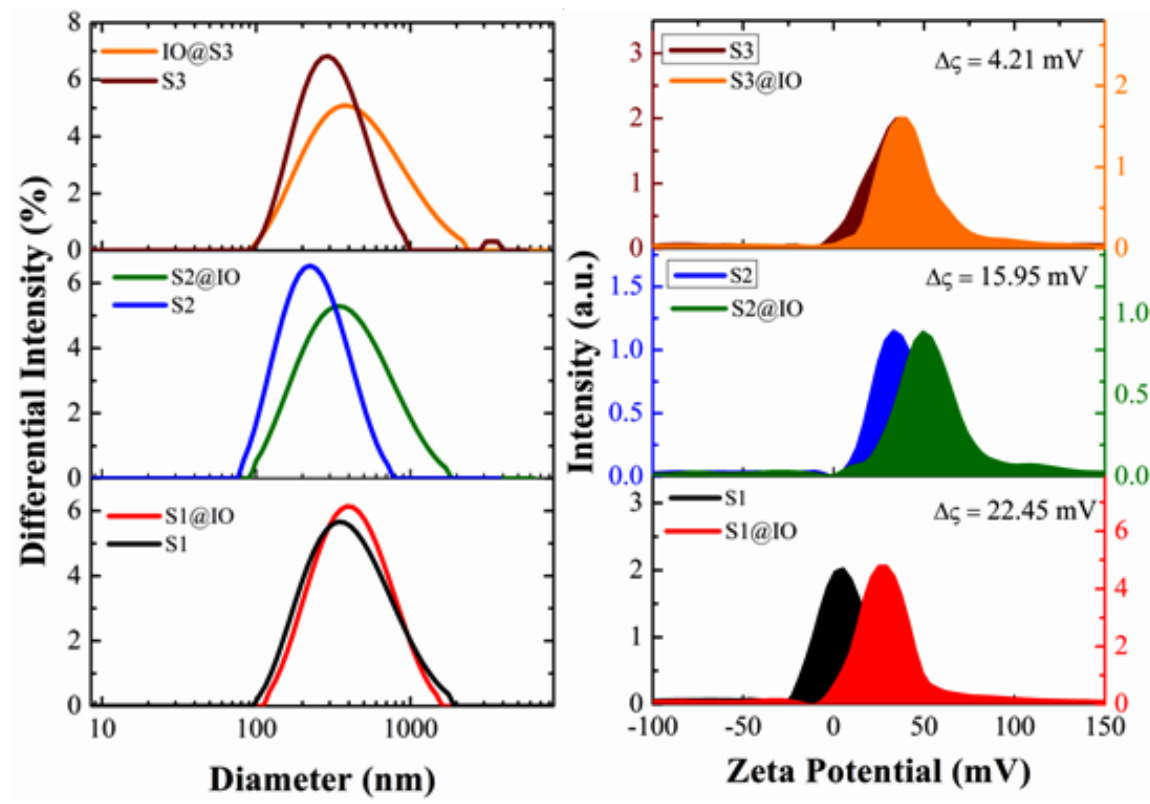

Fig. 5 Comparative size distribution plots from DLS and the comparative Zeta potential plots the three silica samples and silica-nanoparticles conjugate samples. 


\begin{tabular}{|c|c|c|c|c|}
\hline Samples & PDI & $\begin{array}{c}\text { Mean Particle } \\
\text { size }(\mathrm{nm})\end{array}$ & $\begin{array}{c}\text { Zeta Potential } \\
(\mathrm{mV})\end{array}$ & $\begin{array}{c}\text { Difference in Zeta } \\
\text { Potential }(\mathrm{mV})\end{array}$ \\
\hline S1 & 0.684 & $599.79 \pm 24.87$ & $5.02 \pm 0.26$ & 22.45 \\
\cline { 1 - 3 } S1@ IO & 0.664 & $629.41 \pm 9.15$ & $27.47 \pm 0.19$ & \\
\hline S2 & 0.580 & $320.67 \pm 5.69$ & $34.77 \pm 0.25$ & \multirow{2}{*}{15.95} \\
\cline { 1 - 3 } S2@ IO & 0.736 & $620.93 \pm 21.11$ & $50.72 \pm 0.12$ & \\
\cline { 1 - 3 } S3 & 0.588 & $419.19 \pm 11.98$ & $34.33 \pm 0.27$ & \multirow{2}{*}{4.21} \\
\cline { 1 - 3 } S3@ IO & 0.760 & $725.47 \pm 38.29$ & $38.54 \pm 0.38$ & \\
\hline
\end{tabular}

Table 1. Table showing the mean particle sizes, polydispersity indices, Zeta potentials of all of the three silica systems and their respective nanoparticles composite samples.

DLS measurements show a lower value of the polydipersity index for the NPs loaded silica samples than that of the pure silica system (S1). However, for the other two silica samples it is found to be increased after NPs conjugation (shown in the Fig.5 and Table 1), which is due to the non-uniformity of these two composite structures unlike the first sample. The mean hydrodynamic sizes of the silica-nanoparticles composite samples in all the three cases are found to be increased. However, the increments of the hydrodynamic size for the later two samples are noticeable and the reason behind this could be the same, i.e., morphological nonuniformity of these two samples.

On the other hand, the measurement of Zeta potential values shows that the value of the Zeta potential for the NPs loaded system (S1@IO) is increased significantly from that of pure silica system (S1) and this difference for the other two systems decease gradually (shown in the Table 1). This increment of the Zeta potential is due to the enhanced stability of the silica spheres resulted from the uniform enveloping of the CTAB molecules over it during the process of NPs entrapment. Since, this enveloping process for the other two samples is not happening properly and separate aggregations of the nanoparticles are formed outside the silica, hence a smaller difference in Zeta potential values before and after NPs conjugation 
are observed. However, it is noticeable that for the second system (S2), the difference of the Zeta potentials values is not very small, which can be correlated to the partial loading of the particles to silica as mentioned in the earlier discussions.

\section{4 Surface analysis}

XPS has been performed for oleic acid capped $\gamma-\mathrm{Fe}_{2} \mathrm{O}_{3}$ NPs and also for their assembly structure. The Fig.6a shows the complete spectra of the two samples, while the Fig.6b shows the high resolution $\mathrm{Fe} 2 \mathrm{p}, \mathrm{O} 1 \mathrm{~s}$ and $\mathrm{C} 1 \mathrm{~s}$ spectra of both the samples.

(a)
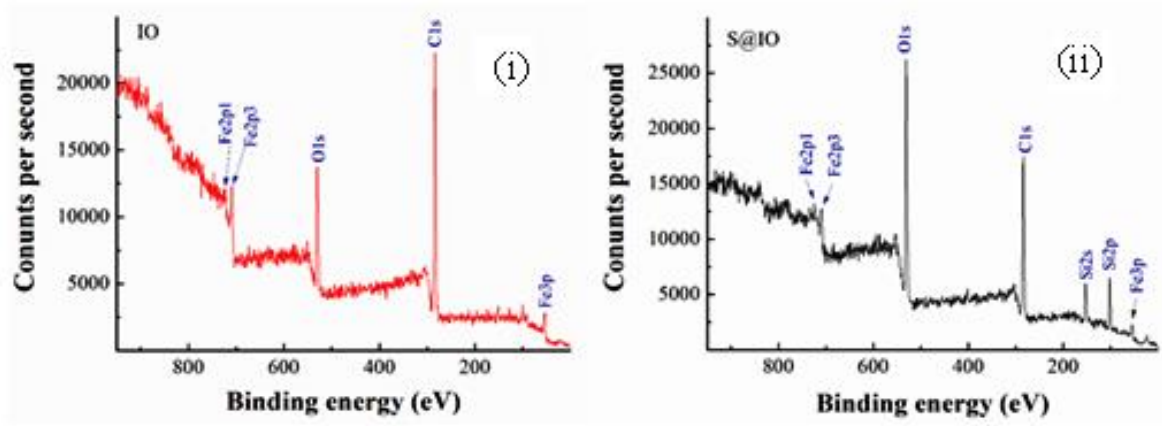

(b)
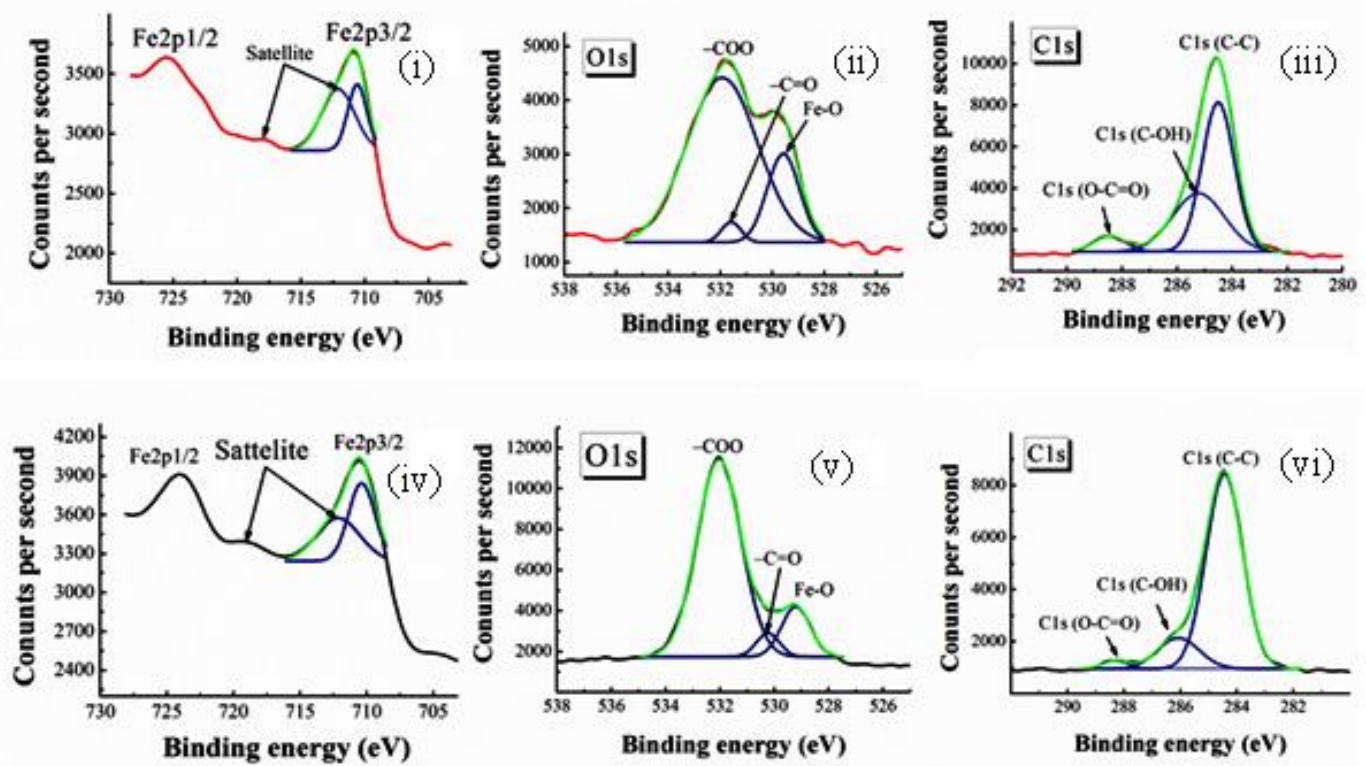

Fig.6 (a) XPS spectra of iron oxide NPs (i) and iron oxide NPs loaded silica spheres (ii); (b) High resolution XPS spectra of Fe2p (i \& iv), O1s (ii \& v) and C1s (iii \& vi) of iron oxide nanoparticles and nanoparticles loaded silica spheres respectively. 
The Fe2p high-resolution XPS spectrum for iron oxide NPs reveals the presence of $\mathrm{Fe} 2 \mathrm{p}_{3 / 2}$ peak at around $710.5 \mathrm{eV}$, along with the satellite peaks at around $712.0 \mathrm{eV}$ and $718.0 \mathrm{eV}$. $\mathrm{Fe} 2 \mathrm{p}_{1 / 2}$ peak at around $725.4 \mathrm{eV}$ gives the evidence of $\mathrm{Fe}^{3+}$ state of $\gamma-\mathrm{Fe}_{2} \mathrm{O}_{3}$ [21]. The spectrum of Fe2p peak for the assembly sample also shows the similar peaks that obtained for the oleic acid capped iron oxide NPs. The high resolution O1s spectra shows three peaks, one at around $529.2 \mathrm{eV}$ resulting from the lattice oxygen of $\gamma-\mathrm{Fe}_{2} \mathrm{O}_{3}$ and other two peaks at around 530.2-531.5 and 532.0 eV raised from mono $(-\mathrm{C}=\mathrm{O})$ and bidentate $(-\mathrm{COO})$ carboxylate oxygen atoms respectively [22]. However, there is clear variation in the relative intensities of these three peaks for the two samples, which could be due to the passivation effect of silica in the assembly sample and also the possibility of changing oleic acid binding configuration to the NPs surface. The high-resolution C1s spectrum of iron oxide NPs gives three peaks at $284.5,285.2$ and $288.5 \mathrm{eV}$, which can be assigned to carbon atoms of aliphatic $-\mathrm{C}-\mathrm{C}$ bonds, $-\mathrm{C}-\mathrm{OH}$ groups and $-\mathrm{COO}-$ groups of oleic acid, respectively [23]. For the assembly sample, all the three peaks have been also observed, but with higher relative peak intensity for $-\mathrm{C}-\mathrm{C}$, which can be due to the contribution of $-\mathrm{C}-\mathrm{C}$ coming from $\mathrm{CTAB}$ molecules. The XPS spectrum of the S@IO sample shows the Si2s and Si2p peaks at around $153.8 \mathrm{eV}$ and $102.8 \mathrm{eV}$ respectively, which are with the good agreement with literature [24]. High resolution spectra of the same, has been shown in the supplementary Fig.s4. 

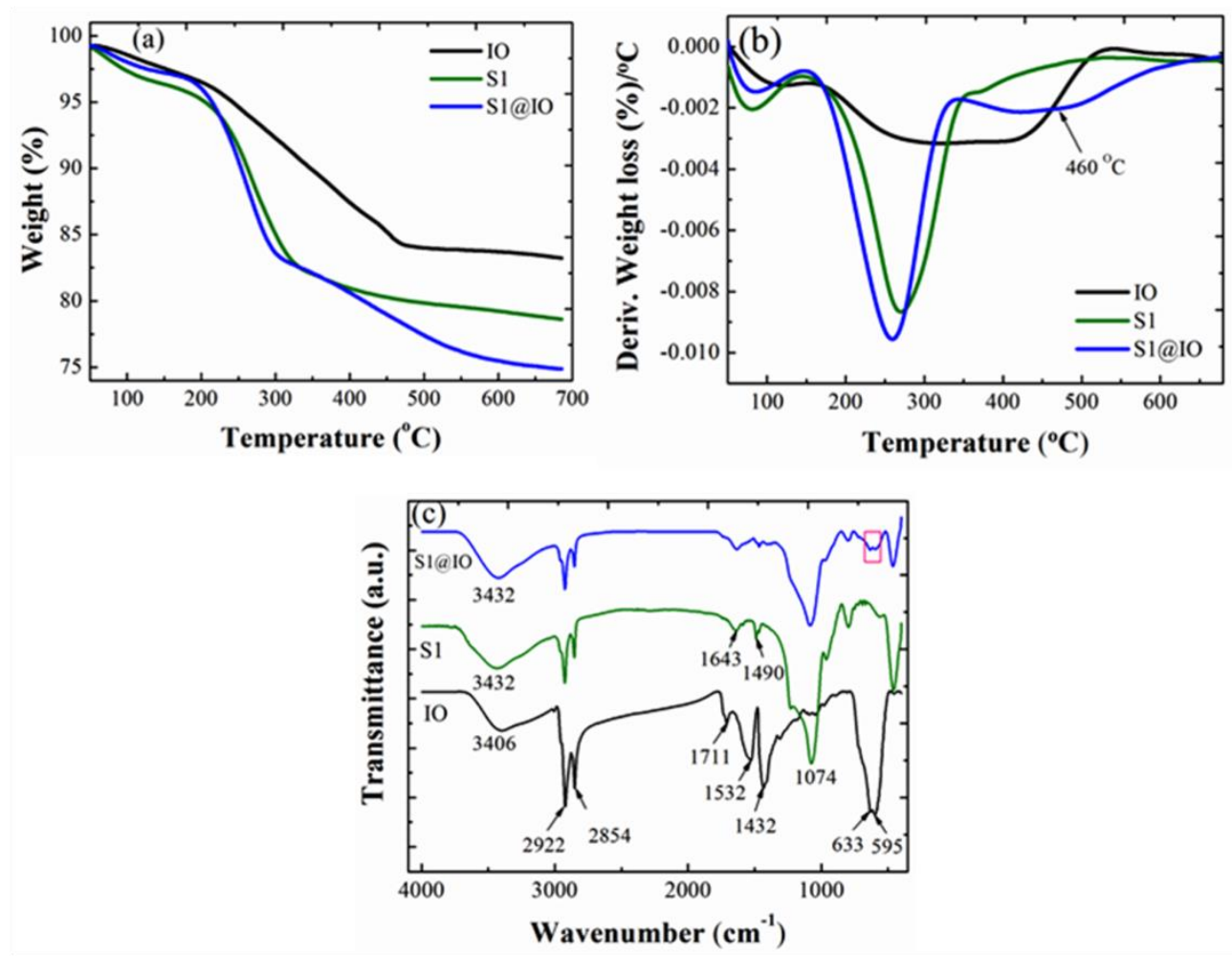

Fig. 7 (a) Comparative TGA; (b) DTG plots of iron oxide nanoparticles, silica spheres and nanoparticles loaded silica spheres and (c) shows the comparative FTIR of iron oxide NPs, silica spheres and NPs loaded silica spheres.

TGA and FTIR characterizations were performed for the maghemite NPs, S1 and S1@IO for further understanding on NPs-silica conjugation characteristics. Fig.7a shows the comparative TGA plots of the iron oxide NPs, pure silica spheres and the nanoparticles loaded silica spheres. The single step weight loss observed for the pure silica sphere system is coming due to the decomposition of the remaining CTAB molecules. However, for the NPs loaded system two distinct weight loss regimes have been observed and the total weight loss was also found higher. DTG plots shown in the Fig. $7 \mathrm{~b}$ further clarifies this two-steps process. The first step weight loss might be due to the decomposition of the CTAB molecules remaining in the silica and the second step weight loss at higher temperature corresponds to the decomposition of oleic acid molecules over the iron oxide NPs. Moreover, it is also observed that the second peak is extended up to higher temperature than that where the 
weight loss of the iron oxide NPs ended. This could be argued as the entrapment of the oleic acid capped NPs inside the pores does not favour the easy degradation of the particles' capping molecules. It is also observed that the inflection point of the weight loss with respect to the degradation of CTAB molecules for the nanoparticles loaded sample is observed little bit at lower temperature and also with higher intensity of the derivative peak. This might be due to the contribution of loosely bound CTAB molecules enveloping the spherical assemblies. Thus, the observed higher weight loss value and the two step weight losses for the silica nanoparticle composite system with the extended second stage weight loss signify the effective NPs loading to the mesoporous silica.

FTIR spectrum of the iron oxide nanoparticles shows the characteristic bands of oleic acid bonding to the nanoparticles surface (shown in the Fig.7c). The prominent peaks at around $633 \mathrm{~cm}^{-1}$ and $595 \mathrm{~cm}^{-1}$ correspond to the $\mathrm{Fe}-\mathrm{O}$ stretching vibration of maghemite phase of the nanoparticles $[25,26]$. The peaks at around $1532 \mathrm{~cm}^{-1}$ and $1432 \mathrm{~cm}^{-1}$ are attributed to the symmetric and asymmetric $\mathrm{COO}^{-}$stretching vibrations, implies that oleic acid molecules are bonded to the nanoparticles through the chelating bonding of the carboxylate group [27]. The peak at around $1711 \mathrm{~cm}^{-1}$ is due to the stretching vibration of $\mathrm{C}=\mathrm{O}$, which signifies that oleic acid molecules are not only bonded bidentately, but also monodentaly over the particles [28]. XPS analysis also gave the same conclusion on this type of binding nature. Similarly, the FTIR spectrum of the pure silica sample has shown all the characteristics peak of silica like, $\mathrm{Si}-\mathrm{O}-\mathrm{Si}$ stretching at around $1074 \mathrm{~cm}^{-1}$, [29] $\mathrm{Si}-\mathrm{OH}$ stretching at around $960 \mathrm{~cm}^{-1},[30] \mathrm{Si}-$ O-Si bending vibration at around $790 \mathrm{~cm}^{-1}$ and $\mathrm{Si}-\mathrm{O}$ bending at around $490 \mathrm{~cm}^{-1}$ [31]. The presence of the CTAB in the pure silica sample is confirmed from the observed peaks at around $2922 \mathrm{~cm}^{-1}, 2854 \mathrm{~cm}^{-1}$ and at $1643 \mathrm{~cm}^{-1}, 1490 \mathrm{~cm}^{-1}$ [32]. The FTIR spectrum of the silica spheres after nanoparticles entrapment shows both the characteristics vibration bands of nanoparticles and silica. It is observed that $\mathrm{Fe}-\mathrm{O}$ stretching bands are visible for the 
composite sample, while the other signature bands of silica have also appeared at the same positions, but with lower intensities. The observed lowering of intensities is obvious due to the entrapment of the nanoparticles inside the pores.

\subsection{Magnetic property studies}
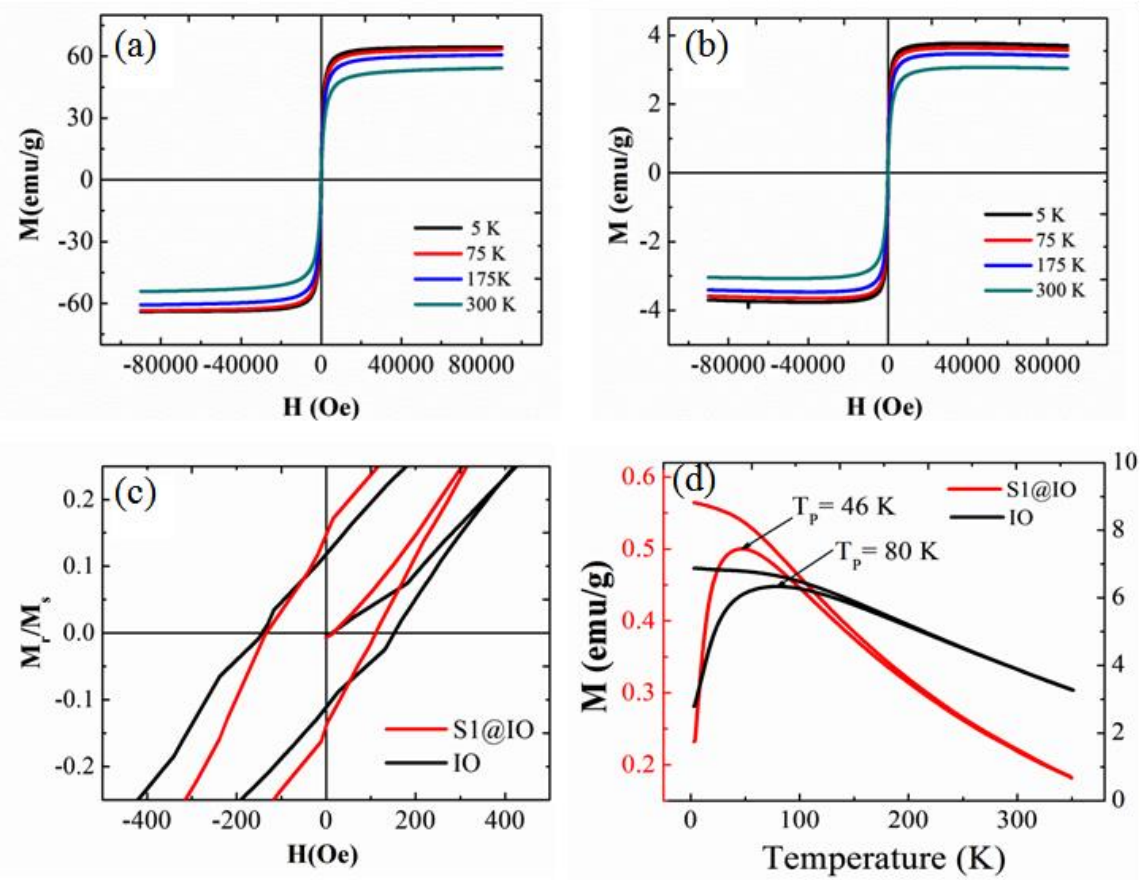

Fig. $8 \mathrm{M}$-H plots of iron oxide nanoparticles (a) and nanoparticles loaded silica spheres (b) at the mentioned temperatures; Fig.c shows the comparative M-H plots (normalized plots) of the two systems at $5 \mathrm{~K}$ and; Fig. d shows the comparative ZFC and FC plots of the two systems in double y plot.

Fig.8 (a) and (b) show the M-H plots of the iron oxide NPs and that of after loading in mesoporous silica spheres respectively. Very small values of coercivity and remanence (close to zero) at $300 \mathrm{~K}$ for both the samples imply the superparamagnetic property of the systems [33]. The oleic acid capped $\gamma-\mathrm{Fe}_{2} \mathrm{O}_{3}$ nanoparticles show the saturation magnetization value around $50 \mathrm{emu} / \mathrm{g}$, which is found smaller than that of the bulk maghemite. This is due to the non-magnetic contribution of the thick oleic acid capping over the particles [34]. It is 
observed that the saturation magnetization $\left(\mathrm{M}_{\mathrm{s}}\right)$ value deceases to $4 \mathrm{emu} / \mathrm{g}$ for the nanoparticles loaded mesoporous silica system. This is obvious due to the lower weight fraction of the nanoparticles in comparison to the host silica materials in the composite system. The value of coercivity $\left(\mathrm{H}_{\mathrm{c}}\right)$ is also obtained smaller for the composite sample than that for the separated iron oxide nanoparticles, while the reduced remanence $\left(\mathrm{M}_{\mathrm{r}} / \mathrm{M}_{\mathrm{s}}\right)$ value shows the opposite observation (shown in Fig.8c).

ZFC and FC measurements for both the samples were measured with probe field 300 Oe (shown in the Fig.8d). The iron oxide nanoparticles system shows the value of blocking temperature of around $80 \mathrm{~K}$. For the assembly system it reduces to $46 \mathrm{~K}$. The observed lowering of the blocking temperature could be related to the average lowering of interparticle dipolar interaction in the assembly system. Though the nanoparticles inside the silica spheres are densely packed, but as a whole there is less interaction among the particles due to the separation among the loaded silica particles. Moreover, it is also observed that the magnetization values above the blocking temperature decrease faster for the assembly system than that for the nanoparticles. This again signifies the less interparticle interaction effect. Thus, it is confirmed that the assembly system has retained superparamagnetic property or even it has shown more towards superparamagnetic property than the iron oxide system. This behaviour would make the system promising for biomedical applications.

\subsection{Biocompability and MRI study}

The MTT (3-(4,5-Dimethylthiazol-2-yl)-2,5-Diphenyltetrazolium Bromide) assay was performed to assess the in vitro cytotoxicity of plant extracts or active compounds using L6 and A549 cell lines. The healthy cells of both the cell lines were harvested in 96 well cell culture grade plates and incubated at the similar conditions. In brief, approximately, 1x104 cells/ml were cultures in DMEM media supplemented with $2 \mathrm{mM}$ L-glutamine, 1mM sodium 
pyruvate, $10 \% \mathrm{FBS}$ and penicillin (100units/ml), streptomycin $(10 \mu \mathrm{g} / \mathrm{ml})$ and allowed to incubate at $37^{\circ} \mathrm{C}$ in a humidified $5 \% \mathrm{CO}_{2}$ environment. After $80 \%$ cell confluence, the cells were treated with different concentrations of each compounds and incubated for $24 \mathrm{hrs}$. After $24 \mathrm{hrs}$ incubation, MTT solution $(5 \mathrm{mg} / \mathrm{ml})$ was added to each well and incubated for a further $4 \mathrm{hrs}$ followed by addition of MTT solvent to dissolve formed formazone complex. The absorbance was taken at $580 \mathrm{~nm}$ and the cell viability was compared to control cells without any treatment.

To observe the cytotoxic effect of the sample, the L6 and A549 cells were treated with different concentrations of it separately for $24 \mathrm{hrs}$. After $24 \mathrm{hrs}$ of the treatment the cells were exposed to MTT solution for $4 \mathrm{hrs}$ forming formazone complex. The absorbance of the dissolved formazone complex revealed that the compound showed the $50 \%$ toxicity towards the cells at concentrations more than $50 \mu \mathrm{g} / \mathrm{ml}$ (shown in Fig.9a). At less than $50 \mu \mathrm{g} / \mathrm{ml}$ concentration, the compound showed moderate toxicity. When the cells treated with $10 \mu \mathrm{g} / \mathrm{ml}$ or less, viability of the cells were found to be more than $75 \%$ in comparison to the control.

(a)

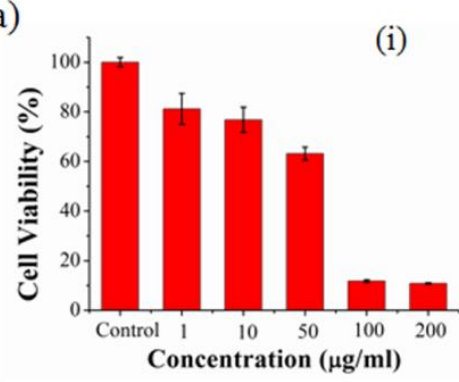

(ii)

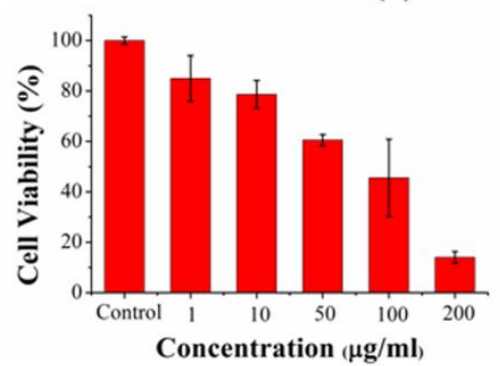

(b)
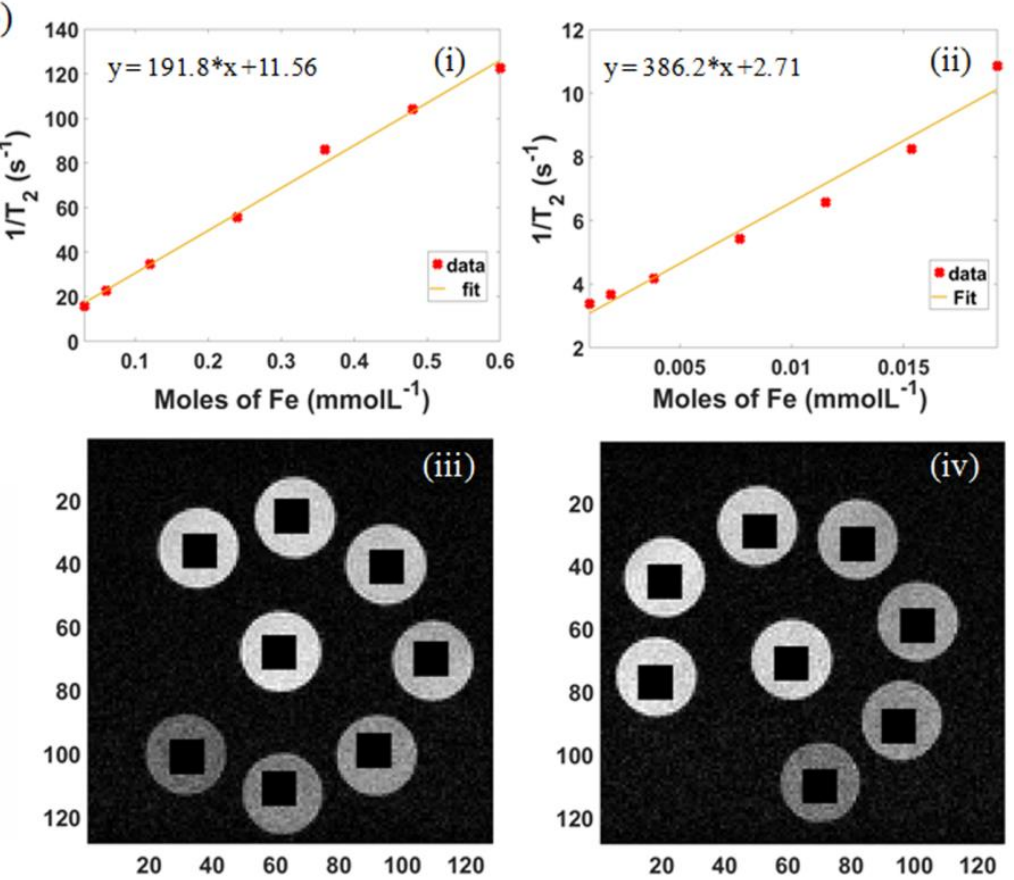
Fig. 9 (a) MTT based cytotoxicity assay for S@IO sample performed on the (i) L6 Skeletal Muscle cell and (ii) A549 Lung cancer cell lines; (b) shows MRI relaxivity plots of $\gamma-\mathrm{Fe}_{2} \mathrm{O}_{3}$ NPs (i) and assembly system (ii) along with the respective phantom images for the two samples, (iii) for $\gamma-\mathrm{Fe}_{2} \mathrm{O}_{3}$ NPs and (iv) assembly system measured at $9.4 \mathrm{~T}$ and ambient temperature. Relaxivity for the NPs was found to be $191.8 \mathrm{~s}^{-1} \mathrm{mM}^{-1}$ and for the assembly it was found to be $386.2 \mathrm{mM}^{-1} \mathrm{~s}^{-1}$

The results in Fig. $9 \mathrm{~b}$ demonstrates that transverse relaxivity of the $\gamma-\mathrm{Fe}_{2} \mathrm{O}_{3} \mathrm{NPs}$ assembly is twice as high as the transverse relaxivity value of the individual NPs. Transverse relaxivity $\left(\mathrm{r}_{2}\right)$ of the individual NPs is $191.8 \mathrm{mM}^{-1} \mathrm{~s}^{-1}$ and for the assembly it is $386.2 \mathrm{mM}^{-1} \mathrm{~s}^{-1}$. Subsequently the values of $r_{2} / r_{1}$ were also calculated which were obtained as 9.59 and 49.20 for the IO nanoparticles and the assembly samples respectively. The $r_{2} / r_{1}$ value for the assembly sample is higher than that of the IO nanoparticles and also higher than the recent reported values, suggesting it as highly efficient negative contrast agents [35-36]. The magnetic moment of NPs is directly related to the transverse relaxation rate following the relation [37].

$$
R_{2}=\frac{1}{T_{2}}=\frac{256 \pi^{2} \gamma^{2}}{405} M_{S}^{2} V^{*} \frac{r^{2}}{D(1+L / r)}
$$

where, $\gamma$ is proton gyromagnetic ratio, $M_{s}$ is the magnetic moment per unit volume of the particle, $V^{*}$ is nanoparticle volume fraction, $r$ is nanoparticle core radius, $D$ is the diffusivity of water molecule at a given temperature and $L$ is the thickness of surface coating. However, this formula does not hold for the assembly. Here a model would require to take into account the exchange rate of water between the interior of the assembly made up by the silica host and the surrounding water as well as the density of NPs within the assembly. It is already seen from the experimental results that the host silica shells have radial pores and the NPs are 
trapped in the pores. The assembly thus provides a region of highly concentrated NPs with relatively good accessibility for water protons as the water protons can traverse through the pores. Due to the high concentration of NPs in the radial pores, water protons are strongly affected by the spatially varying magnetic fields arising from the particles. In contrast, the individual NPs are dispersed uniformly in solution and hence affect only water protons in close proximity. The assembly has a higher $r_{2}$ relaxivity compared to the individual NPs, in analogy to the observation that proton relaxivity increases with the size of magnetic particle [38]. Moreover, this sample has shown higher $r_{2}$ relaxivity value in comparison to the commercially available iron oxide nanoparticles based $r_{2}$ contrast agents like Feridexs and Resovist etc [39]. Also this assembly system has shown superior $r_{2}$ value compared to the other reported clustered iron oxide nanoparticles based systems [40-42].

\section{Conclusion}

In conclusion, our results demonstrate that the dense assembly of maghemite NPs can be developed by packing them in porous silica network through controlled evaporation of solvent. The NPs get entrapped to the pores effectively even after having higher size of the particles than the pore diameter, which is due to the triggering force on the particles to pores as a result of solvent evaporation. However, the nature of pore correlation is one of the key parameter in this mechanism, where it is realized that mesoporosus silica spheres only with straight pores can effectively entrap iron oxide NPs. Magnetic property measurements have showed retaining of superparamagnetic property of the NPs even after dense assembly formation. Also, lowering of interparticle interaction is revealed due to spacing between the individual assemblies even though particles are densely assembled inside the silica spheres. Due to strong collective magnetization and higher accessibility to the water protons through the pores, this secondary nanostructure exhibits significant improvement in MRI transverse relaxivity. This work provides a practical strategy to design high-dimensional ordered 
secondary nanostructure by using ultrafine NPs as basic building blocks for superior efficiency.

\section{Acknowledgments}

The authors acknowledge the UGC-DAE-CSR, Mumbai Centre, India, for providing financial support vide grant no. UDCSR/MUM/AO/CRS-M-186/12/508. PD would like to acknowledge UGC research award grant. The authors also gratefully acknowledge SAIF, NEHU for providing the access to the transmission electron microscopy.

\section{References}

[1] Z. Fu, Y. Xiao, A. Feoktystov, V. Pipich, M. S. Appavou, Y. Su, E. Feng, W. Jin and T. Brückel, Field-induced self-assembly of iron oxide nanoparticles investigated using small-angle neutron scattering, Nanoscale, 8 (2016), pp. 18541-18550.

[2] Y. Tang, Q. Chen, R. Chen, Magnetic field induced controllable self-assembly of maghemite nanocrystals: From 3D arrays to 1D nanochains, App. Surf. Sci., 347 (2015) pp. 202-207.

[3] R. M. Erb, H. S. Son, B. Samanta, V. M. Rotello and B. Y. Benjamin, Magnetic assembly of colloidal superstructures with multiple symmetry, Nature, 457 (2009), pp. 999-1002.

[4] D. K. Jha, M. Shameem, A. B. Patel, A. Kostka, P. Schneider, A. Erbe and P. Deb, Simple synthesis of superparamagnetic magnetite nanoparticles as highly efficient contrast agent, Mater. Lett., 95 (2013), pp.186-189.

[5] E. Peng, F. Wang and J. M. Xue, Nanostructured magnetic nanocomposites as MRI contrast agents, J. Mater.Chem. B, 3 (2015), pp. 2241-2276. 
[6] K. Saikia, S.D. Kaushik, D. Sen, S. Mazumder and P. Deb, Fatty acid as structure directing agent for controlled secondary growth of $\mathrm{CoFe}_{2} \mathrm{O}_{4}$ nanoparticles to achieve mesoscale assemblies: A facile approach for developing hierarchical structures. Appl. Surf. Sci., 379 (2016), pp. 530-539.

[7] A. Blanazs, S. P. Armes and A. J. Ryan, Self-Assembled Block Copolymer Aggregates: From Micelles to Vesicles and their Biological Applications. Macromol. Rapid Commun., 30 (2009), pp. 267-277.

[8] C. E. Smith, A. Shkumatov, S. G. Withers, B. Yang, J. F. Glockner, S. Misra, E. J. Roy, C. Wong, S. C. Zimmerman, and H. Kong, A Polymeric Fastener Can Easily Functionalize Liposome Surfaces with Gadolinium for Enhanced Magnetic Resonance Imaging, ACS Nano, 7 (2013), pp. 9599-9610.

[9] N. Griffete, J. Dechézelles and F. Scheffold, Dense covalent attachment of magnetic iron oxide nanoparticles onto silica particles using a diazonium salt chemistry approach, Chem. Commun., 48 (2012), pp. 11364-11366.

[10] E. Woo, K. M. Ponvel, I.S. Ahn and C.H. Lee, Synthesis of magnetic/silica nanoparticles with a core of magnetic clusters and their application for the immobilization of His-tagged enzymes, J. Mater. Chem., 20 (2010), pp. 1511-1515.

[11] S. Ramesh, I. Felner, Y. Koltypin and A. Gedanken, Reaction Pathways at the Iron-microspherical Silica Interface: Mechanistic Aspects of the Formation of Target Iron Oxide Phases, J. Mater. Res. 15 (2000), pp. 944-950.

[12]M. Ocaña, M. Andrés-Vergés, R. Pozas, and C. J. Serna, Spherical iron/silica nanocomposites from core-shell particles, J. Colloid Interface Sci., 294 (2006), pp. $355-361$. 
[13]Y. Zhu, H. Da, X. Yang and Y. Hu, Preparation and characterization of core-shell monodispersed magnetic silica microspheres, Colloids Surf. A Physicochem. Eng. Aspects, 231(2003), pp. 123-129.

[14]K. Saikia, D. Sen, S. Mazumder and P. Deb, Reassembling nanometric magnetic subunits into secondary nanostructures with controlled interparticle spacing, RSC Adv., 5 (2015), pp. 694-705.

[15]Y. Sun, M. Ma, Y. Zhang and N. Gu, Synthesis of nanometer-size maghemite particles from magnetite, Colloids and Surfaces A: Physicochem. Eng. Aspects, 245 (2004), pp. 15-19.

[16]A. F. Thünemann, D. Schütt, L. Kaufner, U. Pison and H. Möhwald, Maghemite Nanoparticles Protectively Coated with Poly (ethyleneimine) and Poly (ethylene oxide)-block-poly (glutamic acid), Langmuir, 22 (2006), pp. 2351-2357.

[17]I.S. Lyubutin, S.S. Starchikov, T.V. Bukreeva, I.A. Lysenko, S.N. Sulyanov, N. Y. Korotkov, S.S. Rumyantseva, I.V. Marchenko, K.O. Funtov and A.L. Vasiliev, In situ synthesis and characterization of magnetic nanoparticles in shells of biodegradable polyelectrolyte microcapsules, Mater. Sci. Eng. C, 45 (2014), pp. $225-$ 233.

[18]Y. E. Mendili, F. Grasset, N. Randrianantoandro, N. Nerambourg, J. Greneche, J. Bardeau, Improvement of Thermal Stability of Maghemite Nanoparticles Coated with Oleic Acid and Oleylamine Molecules: Investigations under Laser Irradiation. J. Phys. Chem. C, 119 (2015), pp. 10662-10668.

[19]M. Z. Iqbal, X. Ma, T. Chen, L. Zhang, W. Ren, L. Xiang and A. Wu, Silica-coated super-paramagnetic iron oxide nanoparticles (SPIONPs): a new type contrast agent of $\mathrm{T}_{1}$ magnetic resonance imaging (MRI), J. Mater. Chem. B, 3 (2015), pp. 5172-5181. 
[20]H. Xu, Y. Li, Z. Yang, Y. Ding, C. Lu, D. Li and Z. Xu, Preparation of Monodispersed Mesoporous Silica Spheres with Controllable Particle Size Under an Alkaline condition. Int. J. Appl. Cream. Technol., 9 (2012), pp. 1112-1123.

[21]Q. Gao, F. Chen, J. Zhang, G. Hong, J. Ni, X. Wei and D. Wang, The study of novel $\mathrm{Fe}_{3} \mathrm{O}_{4} @ \mathrm{~g}-\mathrm{Fe}_{2} \mathrm{O}_{3}$ core/shell nanomaterials with improved properties, J. Magn. Magn. Mater., 321 (2009), pp.1052-1057.

[22]M. Gogoi, P. Deb, G. Vasan, P. Keil, A. Kostka and A. Erbe, Direct monophasic replacement of fatty acid by DMSA on SPION surface. App. Sur. Sci., 258 (2012), pp. 9685-9691.

[23]D. Wilson and M.A. Langell, XPS analysis of oleylamine/oleic acid capped $\mathrm{Fe}_{3} \mathrm{O}_{4}$ nanoparticles as a function of temperature. App. Surf. Sci., 303 (2014), pp. 6-13.

[24]T. Meng, R. Xie, X. Ju, C. Cheng, S. Wang, P. Li, B. Liang and L. Chu, Nanostructure construction of porous membranes by depositing nanoparticles for enhanced surface testability, J. Memb. Sci., 427 (2013), pp. 63-72.

[25]R. D. Waldron, Infrared spectra of ferrites, Phys. Rev., 99 (1955), pp. 1727-1735.

[26]M. Ma, Y. Zhang, W. Yu, H. Shen, H. Zhang, N. Gu, Preparation and characterization of magnetite nanoparticles coated by amino silane, Colloids Surf. A Physicochem. Eng. Asp., 212 (2003), pp. 219 -226.

[27]A. Hofmann, S. Thierbach, A. Semisch, A. Hartwig, M. Taupitz, E. Rühla and C. Graf, Highly monodisperse water-dispersable iron oxide nanoparticles for biomedical applications. J. Mater. Chem., 20 (2010), pp. 7842-7853.

[28]A. Dreyer, A. Feld, A. Kornowski, E. D. Yilmaz, H. Noei, A. Meyer, T. Krekeler, C. Jiao, A. Stierle, V. Abetz, H. Weller and G.A. Schneider, Organically linked iron oxide nanoparticle supercrystals with exceptional isotropic mechanical properties, Nat. Mater., 15(2016), pp. $522-528$. 
[29]C. Chiang, H. Ishida and J.L. Koenig, The structure of $\gamma$-aminopropyltriethoxysilane on glass surfaces. J. Colloid Interface Sci., 74 (1980), pp. 396-404.

[30]R. Takahashi, S. Sato, T. Sodesawa, M. Kawakita and K. Ogura, High surface-area silica with controlled pore size prepared from nanocomposite of silica and citric acid. J. Phys. Chem. B, 104 (2000), pp. 12184-12191.

[31]M. Gogoi and P. Deb, Magneto-fluorescent hybrid of dye and SPION with ordered and radially distributed porous structures. Appl. Surf. Sci., 298 (2014), pp. 130-136.

[32] Z. Jiang, J. Xie, D. Jiang, X. Wei and M. Chen, Modifiers-assisted formation of nickel nanoparticles and their catalytic application to p-nitrophenol reduction. Cryst. Eng. Comm., 15 (2013), pp. 560-569.

[33]N. D. Thorat, Highly water-dispersible surface-functionalized LSMO nanoparticles for magnetic fluid hyperthermia, New J. Chem., 37 (2013), pp. 2733-2742.

[34]W. Wu, Q. He and C. Jiang, Magnetic Iron Oxide Nanoparticles: Synthesis and Surface Functionalization Strategies, Nanoscale Res. Lett., 3 (2008), pp. 397-415.

[35]M. Khalkhali, K. Rostamizadeh, S. Sadighian, F. Khoeini, M. Naghibi and M. Hamidi, DARU J. Pharm. Sci. 23:45 (2015) pp. 1-12.

[36]S. Khaleghi, F. Rahbarizadeh, D. Ahmadvand, M. Malek, H.R. Madaah Hosseini, Contrast Media Mol. Imaging. 11 (2016) pp. 340-349.

[37]D. Yoo, J. Lee, T. Shin and J. Cheo, Theranostic Magnetic Nanoparticles. Accounts of Chemical Research, 44 (2011), pp. 863-874.

[38] M. R. J. Carroll, R. C. Woodward, M. J. House, W.Y. Teoh, R. Amal, T. L. Hanley and T. G. St Pierre, Experimental validation of proton transverse relaxivity models for superparamagnetic nanoparticle MRI contrast agents, Nanotechnology, 21 (2010), pp. 035103. 
[39]M. Rohrer, H. Bauer, J. Mintorovitch, M. Requardt, H. J. Weinmann, Comparison of Magnetic Properties of MRI Contrast Media Solutions at Different Magnetic Field Strengths, Invest Radiol, 40 (2005), pp. 715-724.

[40]B. A. Larsen, M. A. Haag, N. J. Serkova, K. R. Shroyer and C. R. Stoldt, Controlled aggregation of superparamagnetic iron oxide nanoparticles for the development of molecular magnetic resonance imaging probes, Controlled aggregation of superparamagnetic iron oxide nanoparticles for the development of molecular magnetic resonance imaging probes, Nanotechnology, 19 (2008),pp. 265102.

[41]J.F. Berret, N. Schonbeck, F. Gazeau, D. E. Kharrat, O. Sandre, A. Vacher and M Airiau, Controlled Clustering of Superparamagnetic Nanoparticles Using Block Copolymers: Design of New Contrast Agents for Magnetic Resonance Imaging, J. Am. Chem. Soc., 128 (2006), pp.1755-1761.

[42]S. Balasubramaniam, S. Kayandan, Y. N. Lin, D. F. Kelly, M. J. House, R. C. Woodward, T. G. St. Pierre, J. S. Riffle and R. M. Davis, Toward Design of Magnetic Nanoparticle Clusters Stabilized by Biocompatible Diblock Copolymers for $\mathrm{T}_{2}$ Weighted MRI Contrast, Langmuir, 30 (2014), pp. 1580-1587. 